\title{
Characterization of depolarizing optical media by means of the entropy factor: application to biological tissues
}

\author{
David Pereda Cubián, José Luis Arce Diego, and Raf Rentmeesters
}

\begin{abstract}
Polarized light imaging is a potential tool to obtain an adequate description of the properties of depolarizing media such as biological tissues. In many biomedical applications, for instance, dermatology, ophthalmology, or urology, imaging polarimetry provides a noninvasive diagnosis of a wide range of disease states, and, likewise, it could be applied to the study of internal tissues though the use of endoscopes that use optical fibers. We introduce an algebraic method, based on the Mueller-coherence matrix, for a clearer analysis of the polarization characteristics of depolarizing media via the entropy factor. First-order errors introduced by the measurement system are corrected. Entropy defines three kinds of media according to their depolarizing behavior, and several examples corresponding to each region are shown. The calculation of this factor provides clearer information than that provided by the traditional Mueller matrix in the analysis of biological tissue properties by polarization measurement techniques. (C) 2005 Optical Society of America

OCIS codes: $\quad 000.3860,170.2150,170.3880,170.4580,200.4860,230.5440,260.5430$.
\end{abstract}

\section{Introduction}

Different optical techniques, for instance, spectroscopy, fluorescence, or optical tomography, ${ }^{1-4}$ can be applied to the study and analysis of optical media. Polarimetry is the branch of optical measurements focused on the measurement of polarization properties of samples, and it can be used to analyze the properties of depolarizing optical media.

These media tend to depolarize a large fraction of the incident light, ${ }^{5}$ making the degree of depolarization an interesting parameter to be measured. If a sufficiently significant part of the light remains polarized, this can lead to one's obtaining parameters concerning the relation between incident and emerging polarization states. The study of these parameters can be related to properties of the device under test (DUT). For example, in biological tissues these parameters will be closely related to their biological

D. Pereda Cubian, J. L. Arce Diego (jlarce@teisa.unican.es), and R. Rentmeesters are with the Applied Optics Techniques Group, Department of Electronics Technology, Systems, and Automation Engineering, University of Cantabria, Avenida Castros s/n, 39005 Santander, Spain.

Received 15 March 2004; revised manuscript received 14 July 2004; accepted 20 July 2004

0003-6935/05/030358-08\$15.00/0

(C) 2005 Optical Society of America and physical properties, providing a fundamental way to understand their structure and behavior.

Depolarizing media cannot be characterized by Jones matrix $\mathbf{J},{ }^{6}$ as it can handle only completely polarized light. However, Mueller matrix $\mathbf{M},{ }^{7}$ which works with $4 \times 4$ real matrices, can handle partially polarized light and, consequently, depolarizing media. Moreover, their description using the real Mueller matrix is more interesting for measurements, in that direct intensity measurements can be performed. For obtaining the elements of the Mueller matrix, $m_{i j}$, different measurements must be realized, ${ }^{8}$ and several setups can be discussed. The experimental measurement will provide a $4 \times 4$ Mueller matrix $\mathbf{M}$, which is inevitably measured in the presence of experimental errors and noise, so it is particularly important to define the underlying physically correct Mueller matrix by use of an algorithm to correct the measurement errors introduced by the polarization devices.

Optical properties of the depolarizing media can be described by means of the Mueller-coherence matrix, $\mathbf{C}_{4 \times 4}$, a matrix related to the Mueller matrix and whose properties are equivalent to the well-known $2 \times 2$ coherency matrix $\mathbf{C}_{2 \times 2}$. Optical parameters, such as the so-called entropy factor, can be obtained and give important information on the polarization properties of the optical media. The application of these parameters to biological tissues provides a clear 
interpretation of their polarization characteristics and furnishes extra information closely related to their biological and physical properties, in their analysis.

In this paper the application of the entropy factor obtained from the Mueller-coherence matrix is presented and applied to the characterization of depolarizing media such as biological tissues to obtain clearer information of their polarization behavior than with the Mueller matrix. To obtain a correct characterization of the measurement scheme, we consider the polarization devices nonideal and analyze and correct their influence on the measured values. The scheme of this paper will be as follows. First, the Mueller-coherence matrix and the entropy factor will be defined and described. Next, the measurement method and the errors introduced by optical polariza- factor, one must previously define the Muellercoherence matrix.

The homomorphism $S U(2) \leftrightarrow \mathrm{O}^{+}(3)$ allows connecting the coherence matrix $\mathbf{C}_{\mathbf{2} \times \mathbf{2}}$ and the coefficient Stokes parameters $S_{i}$ characterizing the light ${ }^{8}$ through its decomposition into the Pauli matrices $\left(\sigma_{i}\right)$ basis. ${ }^{13,14}$ In a equivalent way, by means of the homomorphism $S U(4) \leftrightarrow O^{+}(6)$, the Mueller-coherence matrix $\mathbf{C}_{4 \times 4}$ and the Mueller matrix can be connected by use of the Dirac matrices $\left(\eta_{i j}\right)$ basis ${ }^{15}$ :

$$
\mathbf{C}_{\mathbf{2} \times \mathbf{2}}=\frac{1}{2} \sum_{i=0}^{3} S_{i} \sigma_{i} \Leftrightarrow \mathbf{C}_{\mathbf{4} \times \mathbf{4}}=\frac{1}{4} \sum_{i, j=0}^{3} m_{i j} n_{i j} .
$$

The Mueller-coherence elements, $c_{i j}$, and Mueller matrices' elements, $m_{i j}$, are related by the following expressions:

$$
\begin{aligned}
& c_{11}=m_{11}+m_{12}+m_{22}, \\
& c_{12}=m_{13}+m_{23}+i\left(m_{14}+m_{24}\right), \\
& c_{13}=m_{31}+m_{32}-i\left(m_{41}+m_{42}\right), \\
& c_{14}=m_{33}+m_{44}+i\left(m_{34}-m_{43}\right), \\
& c_{21}=m_{13}+m_{23}-i\left(m_{14}+m_{24}\right), \\
& c_{22}=m_{11}-m_{12}-m_{21}-m_{22}, \\
& c_{23}=m_{33}-m_{44}-i\left(m_{34}+m_{43}\right), \\
& c_{24}=m_{31}-m_{32}-i\left(m_{41}-m_{42}\right),
\end{aligned}
$$

$$
\begin{aligned}
& c_{31}=m_{31}+m_{32}+i\left(m_{41}+m_{42}\right), \\
& c_{32}=m_{33}-m_{44}+i\left(m_{34}+m_{43}\right), \\
& c_{33}=m_{11}+m_{12}-m_{21}-m_{22}, \\
& c_{34}=m_{13}-m_{23}+i\left(m_{14}-m_{24}\right), \\
& c_{41}=m_{33}+m_{44}-i\left(m_{34}-m_{43}\right), \\
& c_{42}=m_{31}-m_{32}+i\left(m_{41}-m_{42}\right), \\
& c_{43}=m_{13}-m_{23}-i\left(m_{14}-m_{24}\right), \\
& c_{44}=m_{11}-m_{12}-m_{21}+m_{22} .
\end{aligned}
$$

tion devices (linear polarizer and linear retarder) will be described, showing the mathematical method employed to correct their presence. Then the method of processing the Mueller matrix element values to calculate the entropy factor will be applied to different examples, corresponding to each of the regions in which the entropy values of optical media can be divided. In the first region, as biological tissues do not usually present low values of entropy, an optical device (a linear polarizer) will be described. Afterward, for the second region, a Mueller matrix is extracted from an experimental measurement treating the polarization properties of a biological cell suspension of a cancerous tissue. Finally, for the third region, with high entropy values, an experimental measurement of the characteristics of a human eye by double-pass backscattered light in its interior is described.

\section{Mueller-Coherence Matrix and the Entropy Factor}

Depolarizing media produce not only a variation of the polarization state but also a change of the degree of polarization, making the Mueller matrix the usual method to analyze these media. However, more information can be extracted from these media from parameters such as the entropy factor. To obtain this
From the eigenvalue analysis of the Muellercoherence matrix, additional information can be extracted. The eigenvalues, $\lambda_{i}$, and eigenvectors, $\mathbf{C}_{4 \times 4}$ i, can be easily obtained, as they conform to a set of a maximum of only four terms, as follows:

$$
\mathbf{C}_{4 \times 4}=\lambda_{1} \mathbf{C}_{4 \times 41}+\lambda_{2} \mathbf{C}_{4 \times 42}+\lambda_{3} \mathbf{C}_{4 \times 43}+\lambda_{4} \mathbf{C}_{4 \times 44} .
$$

The number of significant eigenvalues and their values is directly related to the depolarizing characteristics of the media. In polarization-maintaining media, only one eigenvalue is different from zero, providing a direct correspondence between the Jones and the Mueller matrices. On the other hand, in depolarizing devices, some of them take values different from zero, and the concept of entropy $H$ factor is introduced:

$$
\begin{aligned}
H & =-\sum x_{i} \log _{4} x_{i} \Rightarrow x_{i} \\
& =\left|\lambda_{i}\right| / \sum_{j}\left|\lambda_{i}\right|, \quad 0<x_{i}<1 .
\end{aligned}
$$

The entropy factor takes a value from 0 to 1 . An entropy factor 0 corresponds to only one nonzero eigenvalue, that is, media not introducing a depolariza- 
tion and whose polarization state is perfectly known at their output. In the opposite case, with an entropy factor 1 , this corresponds to four identical eigenvalues, and media present an unknown output polarization state. This factor introduces an independent measurement of the polarization behavior of optical media, so it can be properly characterized without any consideration of the input light. According to the value of entropy, three zones can be defined.

\section{A. Small Value of $H$ (between 0 and 0.25 )}

One of the four eigenvalues of $\mathbf{C}_{4 \times 4}$ is much higher than the other three. In this case a dominant eigenvalue exists, and the behavior of the media is only slightly depolarizing. A direct relation between Jones and Mueller matrices can be established from the principal eigenvector.

\section{B. Intermediate Value of $H$ (between 0.25 and 0.8)}

There is no longer a dominant eigenvalue, and the behavior of the principal eigenvector is disturbed. The influence of the other eigenvectors should also be taken into account. Because these depolarizing media cannot be represented by one Jones matrix, deriving the Jones matrix from the dominant eigenvalue is not absolutely meaningful, and its value has to be assumed as a general description of the main behavior but is not a complete and exact description of the DUT.

\section{High Value of $H$ (more than 0.8)}

The former theory is no longer applicable, and the media, with such a high degree of depolarization, cannot be described with the entropy analysis. Other polarization parameters have to be considered to analyze the polarization behavior of the media.

This definition of the entropy factor makes possible the analysis of any optical media. Thus it will be applied to the characterization of biological tissues.

\section{Characterization of Biological Tissues by the Entropy Factor}

As previously described, the entropy factor values can be divided into three regions, in which the polarization properties of the media are strongly different. Different media correspond to each region according to their polarization properties. In this section, this factor will be used to characterize the depolarization properties of biological tissues. First, the measurement scheme and an error-correction method to obtain the exact Mueller matrix of the DUT from the experimental Mueller matrix measured in the presence of errors are also presented.

In the measurement system, which includes both the input and the output polarization devices employed to obtain the Mueller matrix $\mathbf{M}$ of the depolarizing medium (Fig. 1), light coming from an input source is introduced into a polarizer and a quarterwave plate, and its output is sent to a beam splitter (BS). This BS transmits the light on the sample (DUT), focusing it onto the sample through an optical lens. The reflected signal is conducted by the BS to an

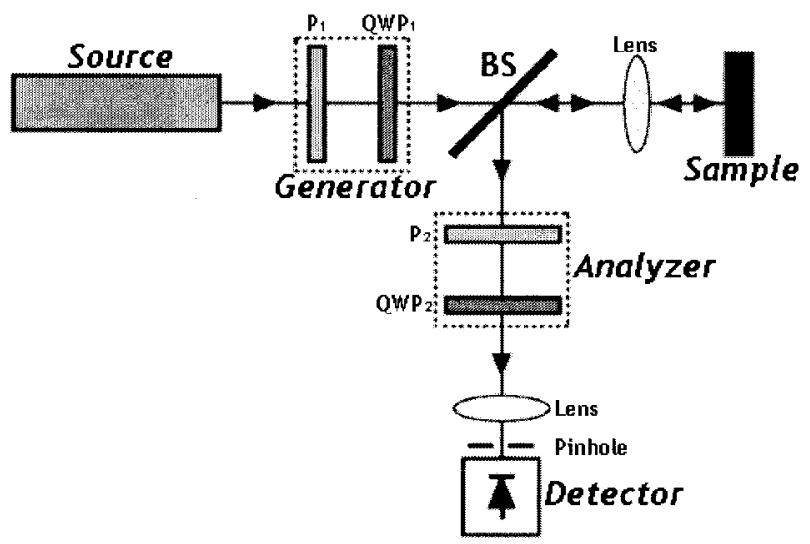

Fig. 1. Measurement system scheme. P, polarizer; QWP, quarterwave plate; BS, beam splitter.

output analyzer and to the detector. The polarizers, quarter-wave plates, and BS are commercial Melles Griot polarization components.

Optical devices introduced into the measurement system to extract the Mueller matrix of an optical media can produce depolarization and introduce error factors in the measurements because of their imperfections. In this paper, no depolarization is considered, and the optical devices are supposed to introduce only first-order errors, in which no relation between the orthogonal components or frequency change is produced. These errors are optical activity $\left(\phi_{P}\right)$, strain $\left(\delta_{P}\right)$, and leakage $\left(\gamma_{P}\right)$ in the linear polarizer and optical activity $\left(\phi_{R}\right)$ and dichroism $\left(\alpha_{R}\right)$ in the linear retarder. ${ }^{16}$ As polarization devices are not supposed to produce modification of the degree of polarization, they could be analyzed by a simple Jones matrix. ${ }^{17}$ However, Mueller matrix results are more general and allows a fast and easy calculation of the whole system to be analyzed, as the DUT are actual depolarizing media that cannot be analyzed by a Jones matrix. Therefore the Mueller matrices with the error factors for the linear polarizers and retarders are calculated, and the influence of these error terms on the elements of the Mueller matrix is analyzed. The values of these elements with the error factors included are shown in detail in Appendix A.

Starting from these equations, an interesting application is to pursue the correct information out of the measured, error-enhanced Mueller matrix. The error parameters of the polarizer and retarder are provided by the manufacturer. In a Mueller matrix measurement, four Stokes columns should be measured for four types of incident light, namely, natural light, horizontal linear polarized light, $45^{\circ}$ with the $x$-axis linear polarized light, and left-handed circular polarized light. 8 These incident polarizations determine for each case the configuration of the input polarization devices, the polarizer, and the first retarder. The configuration of the output polarization devices corresponds to the Mueller matrix element to be measured, so each Mueller matrix element is obtained by means of a certain combination of the input and output polarization devices. 
However, the introduction of errors by the polarizing devices produces a variation of the experimental values from the theoretical ones, in such a way that each measured value results in a combination of different Mueller matrix elements without errors. Sixteen different equations are obtained, one for each Mueller matrix element, from which an equation system with 16 equations and variables is obtained, providing the experimental nonerror values from the measured matrix.

In all the examples the following error factors, corresponding to standard values in polarization commercial devices, ${ }^{16}$ are assumed: $\phi_{P}=\phi_{R}=0.1$, $\gamma_{P}=0.05, \alpha_{R}=0.1$, and $\delta_{P}=0$. Some of the element equations used to obtain the nonerror values of the Mueller matrix, those corresponding to its first column, are shown below:

$$
\begin{aligned}
m_{1,1}{ }^{\text {meas }}= & 1.0227 m_{1,1}{ }^{\text {corr }}-0.1787 m_{2,1}{ }^{\text {corr }}, \\
m_{2,1}{ }^{\text {meas }}= & 0.9974 m_{2,1}{ }^{\text {corr }}, \\
m_{3,1}{ }^{\text {meas }}= & 1.0711 m_{3,1}{ }^{\text {corr }}, \\
m_{4,1}{ }^{\text {meas }}= & -0.0964 m_{1,1}{ }^{\text {corr }}-0.0098 m_{2,1}{ }^{\text {corr }}, \\
& -0.0146 m_{3,1}{ }^{\text {corr }}+0.9691 m_{4,1}{ }^{\text {corr }},
\end{aligned}
$$

where meas (measured) means elements experimentally obtained in the presence of first-order errors and corr (corrected) means those that should have been obtained, without errors and which really correspond to the Mueller matrix elements of the depolarizing media.

Next, the entropy calculation previously described will be applied to three different examples corresponding to the three regions of entropy, to prove the potential of this method. First, as biological tissues do not usually have low values of entropy, an optical device (a linear polarizer) will be used to describe this region. Then two different biological tissues will be used to describe the other two regions, with medium and high values of entropy. In the second region, the biological tissue is a cell suspension of cancerous tissue, and, in the third, a human eye.

\section{A. Entropy under 0.25}

This region corresponds to small values of entropy, that is, nondepolarizing or slightly depolarizing media. Biological tissues are not a good example of media corresponding to this region, as they tend to be highly depolarizing media that depolarize a large fraction of the incident light, with an entropy factor higher than 0.25 in most practical applications. Another medium different from biological tissues has to be used as an example to describe this region of the entropy values.

As an example of a medium with small values of entropy, a simple polarizing device, a horizontal linear polarizer, is analyzed. ${ }^{18} \mathrm{~A}$ red $\mathrm{He}-\mathrm{Ne}$ laser $(\lambda$ $=633 \mathrm{~nm}$ ) is utilized as an input source. Its exper- imental Mueller matrix $\mathbf{M}_{P}{ }^{\text {meas }}$ is

$$
\mathbf{M}_{P}{ }^{\text {meas }}=\left[\begin{array}{crrr}
1 & 1.01 & 0.02 & -0.01 \\
1.03 & 1.04 & -0.01 & 0.01 \\
0.03 & 0.04 & 0.02 & 0.01 \\
-0.02 & -0.02 & 0.02 & -0.02
\end{array}\right] .
$$

We correct this matrix by considering the previously described errors introduced by the polarization devices, obtaining $\mathbf{M}_{P}{ }^{\text {corr }}$ :

$$
\mathbf{M}_{P}{ }^{\text {corr }}=\left[\begin{array}{cccc}
1 & 1 & 0.02 & 0.09 \\
0.92 & 0.92 & 0.03 & 0.09 \\
0.07 & 0.06 & 0.02 & 0.02 \\
0.18 & 0.20 & 0.02 & 0
\end{array}\right] .
$$

From this matrix we calculate the eigenvalues, obtaining a principal eigenvalue much higher than the others and a value of entropy of 0.16 ; thus this is, effectively, a slightly depolarizing device. The equivalent Jones matrix of the principal eigenvector $\mathbf{J}_{P}$ showing the main behavior of the device is

$$
\mathbf{J}_{P}=\left[\begin{array}{cc}
1 & 0.016-0.046 i \\
0.036+0.102 i & 0.005-0.001 i
\end{array}\right] .
$$

This matrix is, as expected, similar to the theoretical matrix of a linear polarizer.

The following examples, in the second and third regions, correspond to media with high depolarizing behavior. Hence a different biological tissue will be used as an example for each region.

\section{B. Entropy between 0.25 and 0.8: Biological Cell Suspension of Cancerous Tissue}

This region comprises the values of depolarizing media, and most biological tissues are included here. The entropy factor is obtained from a biological $10^{8}$ cells $/ \mathrm{cm}^{3}$ suspension of a rat fibroblast cancerous tissue. ${ }^{19}$ The scattering coefficient of this sample is $\mu_{S}{ }^{\prime}=2.2 \mathrm{~cm}^{-1}$, and its transport mean free path $\left(\mathrm{mfp}=1 / \mu_{S}{ }^{\prime}\right)$ is $0.45 \mathrm{~cm}$. Its experimental value is taken at $0.7 \mathrm{mfp}$ and corrected by our considering the errors introduced by the elements of the measurement system. The input source is a green $\mathrm{He}-\mathrm{Ne}$ laser with $\lambda=543 \mathrm{~nm}$. The experimental value of the Mueller matrix $\mathbf{M}_{C}^{\text {meas }}$ is

$$
\mathbf{M}_{C}{ }^{\text {meas }}=\left[\begin{array}{rrrr}
0.92 & 0.52 & -0.14 & -0.03 \\
0.51 & 0.55 & -0.05 & 0.01 \\
0.05 & -0.02 & -0.11 & -0.08 \\
-0.03 & -0.05 & -0.06 & -0.08
\end{array}\right] .
$$

We correct its value by considering the errors introduced by the polarization devices, whose values have been previously described, obtaining $\mathbf{M}_{C}{ }^{\text {corr }}$ : 


$$
\mathbf{M}_{C}{ }^{\text {corr }}=\left[\begin{array}{rrcr}
0.91 & 0.48 & -0.22 & 0.13 \\
0.51 & 0.52 & -0.11 & 0.16 \\
0.05 & -0.01 & -0.108 & -0.07 \\
0.06 & 0.02 & -0.08 & -0.08
\end{array}\right] .
$$

In this case, the correction introduced in the matrix elements is quite small. From $\mathbf{M}_{C}{ }^{\text {corr }}$ the Muellercoherence matrix and the eigenvalues are obtained. The principal eigenvalue takes the value of 1.27 , which means that it is 4.3 times higher than the second one. From these eigenvalues we calculate the entropy, obtaining 0.664 . This demonstrates that this biological tissue, as expected, is depolarizing, and the degree of polarization of the reflected light is strongly modified. The equivalent Jones matrix of the principal eigenvector $\mathbf{J}_{C}$ is an approximation to the main behavior of the device, and its value is

$$
\mathbf{J}_{C}=\left[\begin{array}{cc}
1 & -0.163-0.133 i \\
0.016+0.053 i & -0.088-0.14 i
\end{array}\right] .
$$

This matrix is quite similar to the matrix of a linear polarizer, showing the highly absorbing behavior of this biological tissue.

A deeper analysis of this tissue should be developed, comparing its entropy factor in other situations, that is, when it does not present any cancerous or diseased condition. The value of the entropy factor will probably be modified, so a medical diagnosis could be realized through the study of this factor. Another possible extension of this study would be to consider the depolarizing effects that could take place in the polarization devices, analyzing their influence on the measured values. An intensive and exhaustive study of the value of this factor for different tissues and pathological states should be accomplished to provide a clear and confident tool to medical staff in tissue examination, providing an extra instrument for the development of optical biopsies.

\section{Entropy over 0.8: Human Eye}

Some biological tissues are classified into this third region, in which the high value of the entropy factor shows an extremely high depolarizing behavior. As an example, the entropy factor is applied to the study of the polarization properties of a human eye. In this case, the spatially resolved Mueller matrix $\mathbf{M}_{E}{ }^{\text {meas }}$ of a 2-mm pupil of the right eye of a human being for a certain illumination angle has been obtained, ${ }^{20}$ from a double pass into the eye. A red $\mathrm{He}-\mathrm{Ne}$ laser with $\lambda=633 \mathrm{~nm}$ is used as the input source:

$$
\mathbf{M}_{E}^{\text {meas }}=\left[\begin{array}{crcr}
1 & -0.14 & -0.36 & -0.05 \\
-0.02 & 0.51 & -0.07 & -0.04 \\
-0.3 & -0.35 & 0.5 & -0.06 \\
0.16 & 0.35 & 0.19 & 0.34
\end{array}\right] .
$$

The experimental matrix is corrected with the error parameters previously described, and the matrix

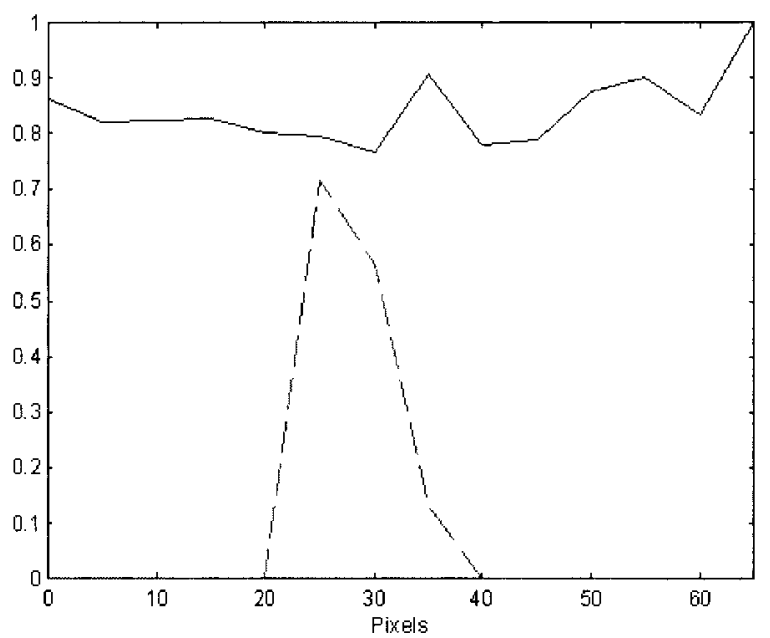

Fig. 2. Entropy factor (continuous curve) and the horizontal-tovertical cross-talk parameter (dashed curve) of the horizontal meridian with the pixels (illumination angle) along the middle of the Mueller image of a human eye.

$\mathbf{M}_{E}{ }^{\text {corr }}$ is obtained:

$$
\mathbf{M}_{E}{ }^{\text {corr }}=\left[\begin{array}{crrr}
0.97 & -0.02 & -0.30 & 0.24 \\
-0.17 & 0.45 & -0.31 & 0.24 \\
-0.4 & -0.04 & 0.49 & 0.19 \\
-0.18 & 0.04 & -0.06 & 0.39
\end{array}\right] .
$$

For this matrix, the entropy factor is very high, 0.813 , so this is an extremely depolarizing medium. The equivalent Jones matrix of the principal eigenvector is no longer meaningful. If the entropy factor is calculated for different pixels corresponding to different angles of illumination, although this matrix provides a good description of the eye, it does not indicate a significant change in its polarization behavior, so the entropy factor is no longer applied (Fig. 2, continuous curve). In this sample, the value of the entropy is found to be extremely high, with only small variations in the central region. This shows a very depolarizing behavior of the eye, with no dependence of the angle.

When the entropy factor does not introduce any significant information, other parameters should be analyzed to obtain an angle-dependent variation of the polarization properties. In this case, the horizontal-to-vertical cross talk, (HVC) is calculated. Because in most applications the relative properties of the DUT are important, the absolute values of the cross talk can also lead to valuable information. The HVC as well as the right-to-left cross talk can be verified. ${ }^{21}$ When the incident light is linearly horizontally polarized, HVC is defined as the ratio

$$
\mathrm{HVC}=\frac{\text { par }- \text { perp }}{\text { par }+ \text { perp }}
$$

When the emerging light is passed through a horizontal polarizer, the measured intensity is par. 
When passed through a vertical polarizer, it produces perp as measured intensity. Right-to-left cross talk is defined analogously. This cross talk can be caused by depolarization or by another polarizing effect of the sample (retardance). The entropy, the cross talk, and the full Mueller matrix are the tools applied in experiments to distinguish different structures or characterize media.

The HVC, in opposition to the entropy factor, suffers a strong variation with the angle in this example (Fig. 2, dashed curve), taking high values in the central region and canceling in the rest. This shows an important polarization-dependent behavior of the central region of the eye, which cannot be observed with the entropy factor. The values obtained for the HVC show the presence of a linear birefringence, as it has been previously described. ${ }^{20}$

Therefore it has been demonstrated that the entropy factor can be an important source of information about the polarization properties of biological tissues, but, in certain cases, this factor can be analyzed together with other parameters such as the cross talk, which provides more complete information than the entropy factor.

\section{Conclusions}

A complete method to analyze the polarizing properties of optical media that modify the degree of polarization has been introduced. This method leads to a transparent analysis of the depolarizing media with the concept of the Mueller-coherence matrix and the entropy factor, together with other parameters such as the cross talk.

From the Mueller matrix $\mathbf{M}$, a $4 \times 4$ coherency matrix $\mathbf{C}_{4 \times 4}$ has been defined, from which polarization parameters such as the entropy factor have been obtained. This factor defines three kinds of media according to their entropy values, directly related to their depolarizing behavior. Different examples of biological tissues, one for each region, have been shown. In all examples, we applied an error-factor correction to obtain the Mueller matrix without errors from the experimental slightly erroneous Mueller matrix, considering the first-order errors introduced by the polarizing devices.

In the first region the entropy values are low, and no biological tissues correspond to it. A horizontal linear polarizer has been studied. In the second region a cell suspension of a cancerous biological tissue has been analyzed, and, in the third region, a human eye has been analyzed. In this final case, as the entropy factor takes a high value and provides little information, other polarization parameters can be used to study the behavior of media and provide some extra information. The horizontal-to-vertical crosstalk has shown a clear variation with the angle in the central regions of the eye.

In conclusion, the utilization of these parameters based on the development of matrix methods for the analysis of depolarizing media could be useful for many interesting biological and medical applications. An exhaustive and complete characterization of dif- ferent biological tissues in different situations should be accomplished in the future to show the potential of this method.

\section{Appendix A}

The values of the Mueller matrix elements with error factors for the linear polarizer and the linear retarder are shown.

These values have been obtained from the corresponding Jones matrices in the presence of errors. ${ }^{16}$ Two methods can be utilized to obtained the equivalent Mueller matrix elements with error factors from the equivalent Jones matrix in the nondepolarizing case. First, Jones and Mueller matrices are related by the expression ${ }^{22}$

$$
\mathbf{M}=\mathbf{U} \cdot\left(\mathbf{J} \otimes \mathbf{J}^{*}\right) \cdot \mathbf{U}^{-1},
$$

where $\mathbf{J}$ is the Jones matrix, $\otimes$ is the Kronecker tensor product, * represents the conjugate, and $\mathbf{U}$ is the Jones-Mueller transformation matrix:

$$
\mathbf{U}=\left[\begin{array}{rrrr}
1 & 0 & 0 & 1 \\
1 & 0 & 0 & -1 \\
0 & 1 & 1 & 0 \\
0 & i & -i & 0
\end{array}\right]
$$

The Mueller matrix can also be obtained from the equivalent Jones matrix by means of the $S U(2) \leftrightarrow O^{+}(3)$ homomorphism. According to this homomorphism, the Mueller matrix elements, $m_{i j}$, are calculated from the Jones matrix by use of the Pauli matrices basis $\left(\sigma_{i}\right)$ and the identity $2 \times 2$ matrix, $\sigma_{0} .{ }^{15}$ Both methods are equivalent and make it possible to obtain the equivalent Mueller matrix elements of any optical device, in this case, a linear polarizer and a linear retarder.

\section{A. Linear Polarizer}

$\theta$ is the angle of the pass plane with the $x$-axis, $\delta$ is the strain error factor $\left(\delta_{P}\right), \varphi$ is the optical activity $\left(\phi_{P}\right)$, and $\gamma$ is the leakage $\left(\gamma_{P}\right)$ :

$$
\begin{aligned}
m_{1,1}= & \frac{1}{8}\left(1+\phi^{2}\right)^{2}(1+\gamma)^{2}+\frac{1}{2} \cos ^{2}(2 \theta)(1-\gamma)^{2} \phi^{2} \\
& +\frac{1}{8}\left(1+\phi^{2}\right)\left[\sin ^{2}(2 \theta)+\delta^{2}\right]-\frac{1}{8}\left(1+\phi^{2}\right)(1-\gamma) \\
& \times \delta \phi \cos (2 \theta)+\frac{1}{8} \cos ^{2}(2 \theta)\left(1-\phi^{2}\right)^{2}(1-\gamma)^{2}, \\
m_{1,2}= & \frac{1}{4} \cos (2 \theta)\left(1-\phi^{4}\right)\left(1-\gamma^{2}\right), \\
m_{1,3}= & \frac{1}{4} \sin (2 \theta)\left(1+\phi^{2}\right)^{2}(1+\gamma), \\
m_{1,4}= & \frac{1}{4} \cos (2 \theta)\left(1-\phi^{4}\right)(1-\gamma) \delta-\frac{1}{2} \cos ^{2}(2 \theta) \phi(1-\gamma)^{2} \\
& \times\left(1-\phi^{2}\right),
\end{aligned}
$$




$$
\begin{aligned}
m_{2,1}= & \frac{1}{4} \cos (2 \theta)\left(1-\phi^{4}\right)\left(1-\gamma^{2}\right), \\
m_{2,2}= & \frac{1}{8}\left(1+\phi^{2}\right)^{2}(1+\gamma)^{2}-\frac{1}{2} \cos ^{2}(2 \theta)(1-\gamma)^{2} \phi^{2} \\
& -\frac{1}{8}\left(1+\phi^{2}\right)\left[\sin ^{2}(2 \theta)+\delta^{2}\right]+\frac{1}{8}\left(1+\phi^{2}\right) \\
& \times(1-\gamma) \delta \phi \cos (2 \theta) \\
& +\frac{1}{8} \cos ^{2}(2 \theta)\left(1-\phi^{2}\right)^{2}(1-\gamma)^{2}, \\
m_{2,3}= & \frac{1}{4} \cos (2 \theta) \sin (2 \theta)\left(1=\phi^{4}\right)(1-\gamma), \\
m_{2,4}= & \frac{1}{4}\left(1+\phi^{2}\right)^{2}(1+\gamma) \delta-\frac{1}{2} \cos (2 \theta) \phi \\
& \times\left(1+\phi^{2}\right)\left(1-\gamma^{2}\right), \\
m_{3,1}= & \frac{1}{4} \sin (2 \theta)\left(1+\phi^{2}\right)^{2}(1+\gamma), \\
m_{3,2}= & \frac{1}{4} \cos (2 \theta) \sin (2 \theta)\left(1-\phi^{4}\right)(1-\gamma), \\
m_{3,3}= & \frac{1}{8}\left(1+\phi^{2}\right)^{2}(1+\gamma)^{2}-\frac{1}{2} \cos ^{2}(2 \theta)(1-\gamma)^{2} \phi^{2} \\
& +\frac{1}{8}\left(1+\phi^{2}\right)\left[\sin ^{2}(2 \theta)+\delta^{2}\right]+\frac{1}{8}\left(1+\phi^{2}\right) \\
& \times(1-\gamma) \delta \phi \cos (2 \theta)-\frac{1}{8} \cos ^{2}(2 \theta) \\
& \times\left(1-\phi^{2}\right)^{2}(1-\gamma)^{2}, \\
m_{3,4}= & 0, \\
m_{4,1}= & \frac{1}{4} \cos (2 \theta)\left(1-\phi^{4}\right)(1-\gamma) \delta-\frac{1}{2} \cos ^{2} \\
& \times(2 \theta) \phi(1-\gamma)^{2}\left(1-\phi^{2}\right), \\
m_{4,2}= & \frac{1}{4}\left(1+\phi^{2}\right)^{2}(1+\gamma) \delta-\frac{1}{2} \cos ^{2}(2 \theta) \phi \\
& \times\left(1+\phi^{2}\right)\left(1-\gamma^{2}\right), \\
m_{4,3}= & 0, \\
m_{4,4}= & \frac{1}{8}\left(1+\phi^{2}\right)^{2}(1+\gamma)^{2}-\frac{1}{2} \cos ^{2}(2 \theta)(1-\gamma)^{2} \phi^{2} \\
& -\frac{1}{8}\left(1+\phi^{2}\right)\left[\sin ^{2}(2 \theta)+\delta^{2}\right]+\frac{1}{8}\left(1+\phi^{2}\right) \\
& \times(1-\gamma) \delta \phi \cos ^{2}(2 \theta) \\
& -\frac{1}{8} \cos ^{2}(2 \theta)\left(1-\phi^{2}\right)^{2}(1-\gamma)^{2} . \\
& \\
& \\
&
\end{aligned}
$$

\section{B. Linear Retarder}

In this device, $\theta$ is the angle of the fast axis with the $x$ axis, $\delta$ is the retardance, $\varphi$ is the optical activity $\left(\phi_{R}\right)$, and $\alpha$ is the dichroism $\left(\alpha_{R}\right)$ :

$$
\begin{aligned}
m_{1,1}= & \frac{1}{8}\left(1+\phi^{2}\right)^{2}[1+\exp (-2 \alpha)+2 \exp (-\alpha) \cos (\delta)] \\
& +\frac{1}{8} \cos ^{2}(2 \theta) \phi^{2}[1+\exp (-2 \alpha) \\
& -2 \exp (-\alpha) \cos (\delta)]+\frac{1}{8} \cos ^{2}(2 \theta)\left(1-\phi^{2}\right)^{2} \\
& +\sin ^{2}(2 \theta)\left(1+\phi^{2}\right)[1+\exp (-2 \alpha) \\
& -2 \exp (-\alpha) \cos (\delta)] \\
m_{1,2}= & \frac{1}{4} \cos (2 \theta)\left(1-\phi^{4}\right)[1-\exp (-2 \alpha)] \\
& +\frac{1}{4} \sin (2 \theta) \cos (2 \theta) \phi\left(1+\phi^{2}\right)[1-\exp (-2 \alpha) \\
& -2 \exp (\alpha) \cos (\delta)], \\
m_{1,3}= & \frac{1}{4} \sin (2 \theta)\left(1+\phi^{2}\right)^{2}[1-\exp (-2 \alpha)] \\
& -\frac{1}{4} \cos { }^{2}(2 \theta) \phi\left(1+\phi^{2}\right)[1+\exp (-2 \alpha) \\
& -2 \exp (-\alpha) \cos (\delta)], \\
m_{1,4}= & \frac{1}{2} \cos (2 \theta) \phi\left(1+\phi^{2}\right) \exp (-\alpha) \cos (\delta),
\end{aligned}
$$

$$
\begin{aligned}
m_{2,1}= & \frac{1}{4} \cos (2 \theta)\left(1-\phi^{4}\right)[1-\exp (-2 \alpha)] \\
& -\frac{1}{4} \sin (2 \theta) \cos (2 \theta) \phi\left(1+\phi^{2}\right)[1+\exp (-2 \alpha) \\
& -2 \exp (-\alpha) \cos (\delta)] \\
m_{2,2}= & \frac{1}{8}\left(1+\phi^{2}\right)^{2}[1+\exp (-2 \alpha)+2 \exp (-\alpha) \cos (\delta)] \\
& -\frac{1}{8} \cos ^{2}(2 \theta) \phi^{2}[1+\exp (-2 \alpha) \\
& -2 \exp (-\alpha) \cos (\delta)], \\
m_{2,3}= & \frac{1}{4} \sin (2 \theta) \cos (2 \theta)\left(1-\phi^{4}\right)[1+\exp (-2 \alpha) \\
& -2 \exp (-\alpha) \cos (\delta)] \\
& -\frac{1}{4} \cos (2 \theta) \phi\left(1+\phi^{2}\right)[1-\exp (-2 \alpha)] \\
m_{2,4}= & -\frac{1}{2} \sin (2 \theta) \exp (-\alpha)\left(1+\phi^{2}\right)^{2} \sin (\delta), \\
m_{3,1}= & \frac{1}{4} \sin (2 \theta)\left(1+\phi^{2}\right)^{2}[1-\exp (-2 \alpha)] \\
& +\frac{1}{4} \cos { }^{2}(2 \theta) \phi\left(1+\phi^{2}\right)[1+\exp (-2 \alpha) \\
& -2 \exp (-\alpha) \cos (\delta)], \\
m_{3,2}= & \frac{1}{4} \sin (2 \theta) \cos (2 \theta)\left(1-\phi^{4}\right)[1+\exp (-2 \alpha) \\
& -2 \exp (-\alpha) \cos (\delta)]+\frac{1}{4} \cos (2 \theta) \phi\left(1+\phi^{2}\right) \\
& \times[1-\exp (-2 \alpha)], \\
m_{3,3}= & \frac{1}{8}\left(1+\phi^{2}\right)^{2}[1+\exp (-2 \alpha)+2 \exp (-2 \alpha) \cos (\delta)] \\
& -\frac{1}{8} \cos { }^{2}(2 \theta) \phi^{2}[1+\exp (-2 \alpha) \\
& -2 \exp (-2 \alpha) \cos (\delta)]-\frac{1}{8} \cos { }^{2}(2 \theta)\left(1+\phi^{2}\right)^{2} \\
& -\sin { }^{2}(2 \theta)\left(1+\phi^{2}\right)[1+\exp (-2 \alpha) \\
& -2 \exp (-\alpha) \cos (\delta)], \\
m_{3,4}= & \frac{1}{2} \cos (2 \theta) \exp (-\alpha) \sin (\delta)\left(1+\phi^{4}\right), \\
m_{4,1}= & \frac{1}{2} \cos (2 \theta) \phi\left(1+\phi^{2}\right)^{2} \exp (-\alpha) \cos (\delta), \\
m_{4,2}= & \frac{1}{2} \sin (2 \theta) \exp (-\alpha)\left(1+\phi^{2}\right)^{2} \sin (\delta), \\
m_{4,3}= & -\frac{1}{2} \cos (2 \theta) \exp (-\alpha) \sin (\delta)\left(1+\phi^{4}\right), \\
m_{4,4}= & \frac{1}{8}\left(1+\phi^{2}\right)^{2}[1+\exp (-2 \alpha)+2 \exp (-\alpha) \cos (\delta)] \\
& +\frac{1}{8} \cos { }^{2}(2 \theta) \phi^{2}[1+\exp (-2 \alpha) \\
& -2 \exp (-\alpha) \cos (\delta)]-\frac{1}{8} \cos { }^{2}(2 \theta)\left(1+\phi^{2}\right)^{2} \\
& -2 \theta)\left(1+\phi^{2}\right)[1+\exp (-2 \alpha) \\
& \exp (\delta)] \\
& \\
&
\end{aligned}
$$

\section{References}

1. T. Vo-Dich, Handbook on Biomedical Photonics (CRC Press, Boca Raton, United States, 2003).

2. V. Tuchin, Tissue Optics: Light Scattering Methods and Instruments for Medical Diagnosis Vol. TT38 of Tutorial Texts in Optical Engineering (SPIE, Bellingham, Wash., 2000).

3. G. A. Wagnieres, W. M. Star, and B. C. Wilson, "In vivo fluorescence spectroscopy and imaging for oncological applications," Photochem. Photobiol. 68, 603-632 (1998).

4. D. Huang, E. A. Swanson, C. P. Lin, J. S. Shuman, W. G. Stinson, W. Chang, M. R. Hee, T. Flotte, K. Gregory, C. A. Puliafito, and J. G. Fujimoto, "Optical coherence tomography," Science 254, 1178-1181 (1991).

5. W. F. Cheong, S. A. Prahl, and A. J. Welch, "A review on optical properties of biomedical tissues," IEEE Quantum Electron. 26, 2166-2185 (1990). 
6. R. C. Jones, "A new calculus for the treatment of optical systems," J. Opt. Soc. Am. A 31, 488-503 (1941).

7. R. M. A. Azzam and N. M. Bashara, Ellipsometry and Polarized Light (North-Holland, Amsterdam, 1977).

8. A. Gerard and J. M. Burch, Introduction to Matrix Methods in Optics (Wiley, London, 1975).

9. J. Lee, J. Koh and R. W. Collins, "Multichannel Mueller matrix ellipsometer for real-time spectroscopy of anisotropic surfaces and films," Opt. Lett. 25, 1573-1575 (2000).

10. J. M. Schmitt, "Optical coherence tomography (OCT): a review," IEEE J. Sel. Top. Quantum Electron. 5, 1205-1215 (1999).

11. S. Jiao and L. V. Wang, "Jones-matrix imaging of biological tissues with quadruple-channel optical coherence tomography," J. Biomed. Opt. 7, 350-358 (2002).

12. P. Y. Han, G. C. Cho, and X. C. Zhang, "Time-domain transillumination of biological tissues with terahertz pulses," Opt. Lett. 25, 242-244 (2000).

13. D. Bohm, Quantum Theory (Dover, New York, 1989).

14. D. Pereda Cubián, C. Vlcek, J. L. Arce Diego, and Z. Zaoralek, "Variation of the Pauli matrices coefficients in Nd-doped fibers subjected to a magnetic field," in Proceedings of IEEE Lasers and Electro-Optics Society, 2001, (IEEE, Piscataway, N. J., 2001), pp. 823-824.

15. S. R. Cloude, "Group theory and polarization algebra," Optik 75, 26-36 (1986).

16. S. Li, "Jones-matrix analysis with Pauli matrices: application to ellipsometry,” J. Opt. Soc. Am. A 17, 920-926 (2000).
17. D. Pereda Cubián and J. L. Arce Diego, "Variation of the Pauli matrices coefficients in a PCA system under non-desired effects for ellipsometric applications," in Proceedings of IEEE Workshop on Fiber and Optical Passive Components (IEEE, Piscataway, N. J., 2002), pp. 182-188.

18. J. M. Bueno, "Polarimetry using liquid-crystal variable retarders: theory and calibration,” J. Opt. A Pure Appl. Opt. 2, 216$222(2000)$.

19. A. H. Hielscher, A. A. Eick, J. R. Mourant, D. S. Shen, J. P. Freyer, and I. J. Bigio, "Diffuse backscattering Mueller matrices of highly scattering media," Opt. Express 1, 441-453 (1997).

20. J. M. Bueno, "Measurement of parameters of polarization in the living human eye using imaging polarimetry," Vision Res. 40, 3791-3799 (2000).

21. S. L. Jacques, A. Gutsche, J. Schwartz, L. Wang, and F. K. Tittel, "Video reflectometry to extract optical properties of tissue in in vivo," in Medical Optical Tomography: Functional Imaging and Monitoring, G. Mueller, B. Chance, R. R. Alfano, S. R. Arridge, J. Beuthan, E. Gratton, M. Kaschke, B. R. Masters, S. Svanberg, and P. van der Zee, eds. (SPIE, Bellingham, Wash., 1992) pp. 211-226.

22. F. Le Roy-Brehonnet and B. La Jeune, "Utilization of Mueller matrix formalism to obtain optical targets depolarization and polarization properties," Prog. Quantum Electron. 21, 109151 (1997). 